\title{
TIE POINT GENERATION IN HYPERSPECTRAL CUBES FOR ORIENTATION WITH POLYNOMIAL MODELS
}

\author{
A. Berveglieri ${ }^{1, *}$, A. M. G. Tommaselli ${ }^{2}$, L. D. Santos ${ }^{3}$ \\ ${ }^{1}$ Department of Statistics, São Paulo State University, Pres. Prudente, S.P., Brazil - a.berveglieri@unesp.br \\ ${ }^{2}$ Department of Cartography, São Paulo State University, Pres. Prudente, S.P., Brazil - a.tommaselli@unesp.br \\ ${ }^{3}$ Graduate Student, São Paulo State University, Pres. Prudente, S.P., Brazil - dias_lucas@hotmail.com
}

\section{Commission II}

KEY WORDS: Bundle Adjustment, Photogrammetry, Hyperspectral camera

\begin{abstract}
:
This paper presents a technique for tie point generation in hyperspectral images collected by a camera with time-sequential principle for band acquisition (i.e., non-synchronized bands). In mobile applications, each band is acquired at a different time, which generates different camera positions and attitude angles. Due to the large number of bands, a bundle adjustment with polynomial models can be applied to sample bands and then, EOP of other bands are interpolated. The determination of homologue points in all sample bands is required to ensure geometric robustness. A procedure was developed to extract tie points from a reference band which were then transferred to the other sample bands. The technique uses a Helmert geometric transformation combined with majority voting to estimate point transfer functions, followed by area-based matching. Experiments with image orientation were conducted to apply and assess the technique. The tests showed an increase in height discrepancy when tie points are not located in all sample bands highlighting the relevance of the proposed filtering. The accuracy of the technique achieved less than 1 GSD in planimetry and 2 GSD in altimetry using the tie points with the maximum number of rays. Thus, the polynomial approach enables interpolation of other bands according to the parameters of the polynomial function.
\end{abstract}

\section{INTRODUCTION}

Lightweight hyperspectral cameras, embedded in unmanned aerial vehicles (UAV), have enabled many applications due to the acquisition of data at high spectral, spatial, and temporal resolution with lower costs when compared to other types of traditional technologies. For example, tree species, plant health or some types of diseases can be detected in forestry or agricultural applications.

Several studies and applications have been developed with hyperspectral cameras aboard UAVs. For example, Aasen et al.(2015) implemented a method for obtaining 3D hyperspectral data from snapshot cameras for vegetation monitoring. Oliveira et al. (2019) developed a technique to generate hyperspectral digital surface models for complex cover studies such as forests. Honkavaara et al. (2017) proposed a band registration technique based on a hyperspectral tunable filter for forest applications. Moriya et al. (2017) performed a study on sugarcane virus detection from hyperspectral images. Jakob et al. (2017) used hyperspectral imaging for mineral exploration. Thus, hyperspectral sensors in UAV platforms have many types of application that can produce good results.

In this context, a type of camera with wide application possibilities is the Rikola hyperspectral camera (Senop Ltd., 2017), which is assembled with two sensors in frame format and allows the image orientation by photogrammetric techniques. The images can be collected with suitable overlap and enable $3 \mathrm{D}$ reconstruction, in which radiometric and geometric analysis can be made for more detailed studies on the objects. This camera uses a (Fabry-Perot interferometer) FPI-based technology that acquires unsynchronized spectral bands, that is, it uses a time-sequential mode to produce the spectral bands. Thus, when collecting images in mobile applications, each band is acquired at a different time, which generates different camera position and attitude for each band of the hypercube (as depicted in Figures 1a,b).

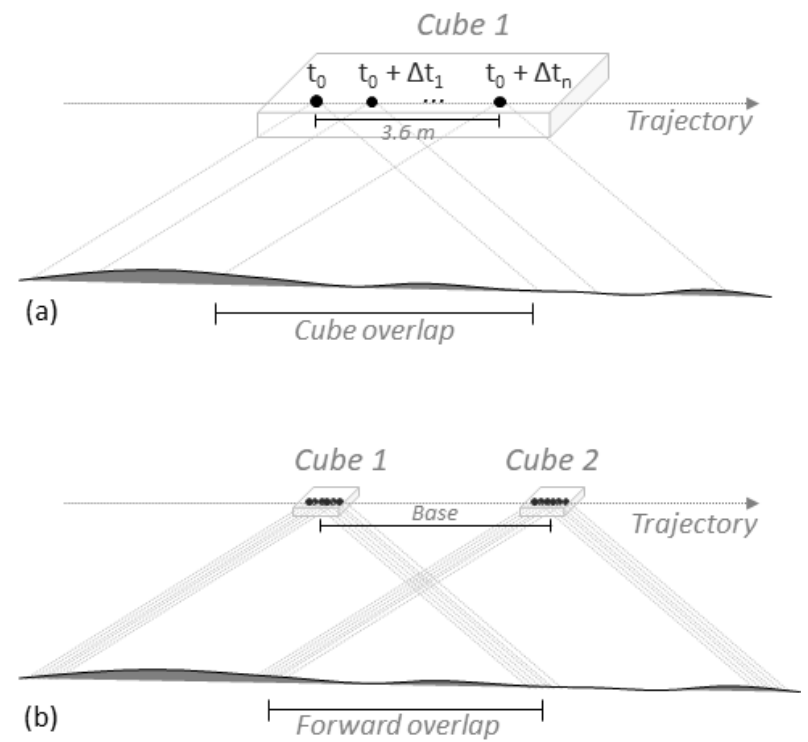

Figure 1. Band displacement when collecting images with the platform in movement in the flight direction. (a) Band displacement as a function of time in a cube. (b) Relationship between cubes.

* Corresponding author 
Due to the large number of images generated by the acquisition of hypercubes, instead of computing a set of six exterior orientation parameters (EOPs) for each image band, an alternative solution is to use bundle adjustment with polynomial models. From some sample bands, polynomial parameters describing the camera trajectory are estimated and then the EOPs of the other spectral bands within the hypercubes can be interpolated (Berveglieri et al., 2017, 2019a) . For this task, tie points are essential to ensure the robustness and accuracy of the polynomial technique like in conventional bundle adjustment (BA).

However, the major problem is the radiometric variation of the bands, which complicates the determination of the same point in multiple bands. Then, a procedure was performed to ensure automatic extraction of tie points in all sample bands, considering the maximum number of rays.

\section{MATERIAL AND METHOD}

\subsection{Material}

The FPI camera used in this study (Figure 2) was developed by the VTT Technical Reserch Centre of Finland (Senop Ltd., 2017) and its main technical features are presented in Table 1.

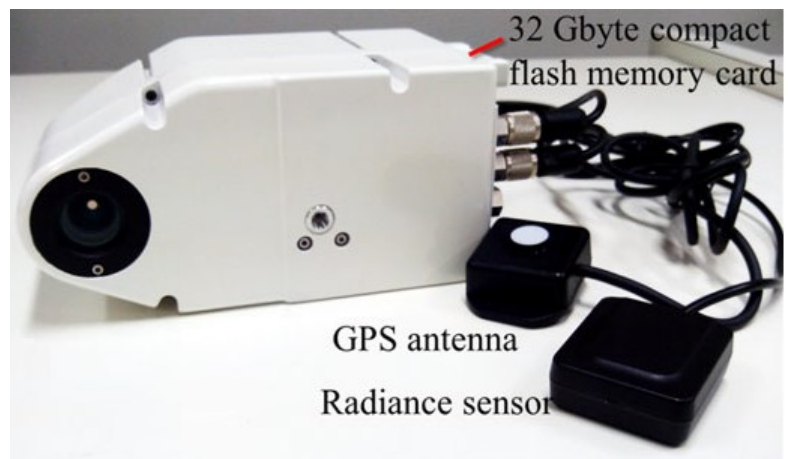

Figure 2. FPI camera used in this study.

\begin{tabular}{|l|l|}
\hline Model & Rikola (FPI2015) \\
\hline Focal length & $9 \mathrm{~mm}$ \\
\hline Image frame dimension & $1017 \times 648$ pixels \\
\hline Pixel size & $5.5 \mu \mathrm{m}$ \\
\hline Spectral range & $500-900 \mathrm{~nm}$ \\
\hline Two CMOS sensors & $(500-636 \mathrm{~nm})$ and $(650-900 \mathrm{~nm})$ \\
\hline
\end{tabular}

Table 1. Technical features of the FPI camera.

The images captured by the camera are hyperspectral bands generated within the $500-900 \mathrm{~nm}$ spectrum. A radiance sensor and a GPS (navigation accuracy) receiver are connected to the camera. The internal mechanisms of the FPI camera use a variable air gap between two parallel reflective surfaces. Multiple light ray reflections at various air gaps produce different wavelengths. Each band is collected with the FPI tuned to a specific interval. The hyperspectral cube is formed by the time-sequential band acquisition. As the FPI camera uses tuneable filters, the number of bands is set by an operator according to the type of application.

In a conventional photogrammetric aerial survey, the platform movement follows an approximately linear trajectory, which results in band displacements in the flight direction. Such effects cause different positions and attitude angles for each band. The misalignment between the two camera sensors and vibrations during image acquisition also contribute to the band displacement. However, this displacement in each hypercube can be modelled by polynomials as function of time, as presented by Berveglieri et al. (2019). To solve this problem, corresponding points are required to perform procedures of image orientation or co-registration.

\subsection{Method}

The determination of homologue points in sample bands is required for the estimation of the polynomial parameters in the modified bundle adjustment. An important requirement is that corresponding tie points are determined in all sample bands, including hypercubes in forward and side directions (adjacent strips). If tie points are only extracted within the same cube, the quality of the result will be affected due to the short baseline. Therefore, tie points must appear in several hypercubes with suitable geometry. In addition, it is well known that suitable geometric distribution of points in images improve the image orientation procedure in bundle adjustment.

The technique for tie point generation is based on a previous work (Berveglieri and Tommaselli, 2019) and can be described in four main steps as detailed below:

1. SIFT keypoints (Lowe, 2004): distinct points are extracted from four sample spectral bands in each hypercube. In this case, we have adopted the first, last, and two intermediate bands of the image sequence.

2. Estimation of point transfer functions between spectral bands: the first band is used as a reference and then the image matching is performed with the SIFT keypoints to produce pairs of matches. This procedure is done using the reference band with the $1^{\text {st }}$ intermediate band; the reference band with the $2^{\text {nd }}$ intermediate band; and the reference band with the last band. In each image pair, the most reliable match pairs are selected using a 2D Helmert geometric transformation combined with a majority voting technique to define a point transfer function between spectral bands. The 2D Helmert geometric transformation (Mikhail et al., 2001) is composed of four parameters (scale $\lambda$, rotation $\theta$ and translations Tx and Ty). As scale and rotation can be approximately pre-defined by the flight plan, these parameters can be considered fixed. Thus, only the two shifts parameters (Tx and Ty) need to be calculated, as presented in Equation 1, in which ( $\left.\mathrm{x}_{1}, \mathrm{y}_{1}\right)$ are coordinates of the keypoint in the image 1 and $\left(\mathrm{x}_{2}, \mathrm{y}_{2}\right)$ are coordinates of the corresponding keypoint in the image 2 .

$$
\left[\begin{array}{l}
T_{x} \\
T_{y}
\end{array}\right]=\left[\begin{array}{l}
x_{2} \\
y_{2}
\end{array}\right]-\lambda\left[\begin{array}{cc}
\cos \theta & -\sin \theta \\
\sin \theta & \cos \theta
\end{array}\right]\left[\begin{array}{l}
x_{1} \\
y_{1}
\end{array}\right]
$$

The results of each pair Tx and Ty are computed in a normalized accumulator matrix, which represents a space discretized in pixels, as depicted in Figure 3. Positions in the matrix work as bins representing range of values. Each coordinate pair (Tx, Ty) of the Equation 1 adds one vote to a matrix position. The position with the largest number of votes indicates which matches should be selected. If there are two positions with the majority of votes, then both 
indicate which matches should be selected. The extracted matches are then used to estimate the four parameters of the Helmert transformation by least-squares method. In this adjustment, if any match still has a residual above a predefined threshold, then the match pair is removed. Finally, a point transfer function between spectral bands is obtained. This approach works well within a hypercube because the parallax effect is very small.
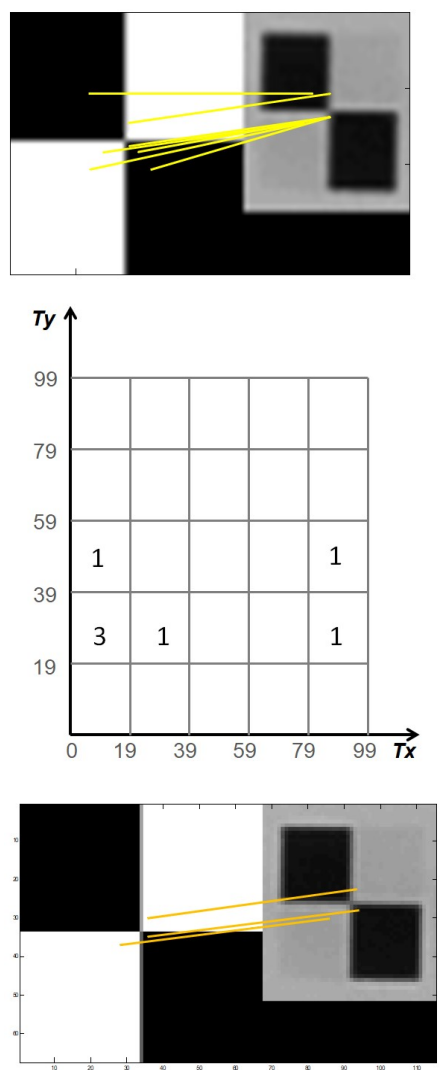

Figure 3. Computation of the pairs (Tx, Ty) in the accumulator matrix to select reliable matches.

3. Extraction of points in sub-regions in the reference band: the reference band is divided into sub-regions to extract well-distributed points in the image, which will be tie points, if they are matched in all sample bands.

4. Point transfer: from the reference band, the distinct points (from step 3) are transferred to the corresponding band by the function estimated in step 2 . In the match band, a window is opened, and the tie point is matched by areabased correlation with sub-pixel refinement by least squares. This step ensures that the distinct points of the reference band are located in all sample bands, including the adjacent hypercubes.

Thus, a set of tie points is generated. Such points appearing in all sample bands ensure a good geometry for estimating the EOPs of the sample bands, which follow the mathematical model defined by the polynomial technique. Consequently, the other intermediate bands can be interpolated by the polynomials resulting from the bundle adjustment, as performed by Berveglieri et al. (2019).

\section{EXPERIMENT AND RESULT}

\subsection{Image acquisition}

Experiments with image orientation were performed to apply and assess the automatic tie point generation. Firstly, the FPI camera was set to collect 25 bands per cube. A camera selfcalibrating procedure (Kenefick et al., 1972) was performed in a terrestrial calibration field to estimate the inner orientation parameters (IOPs): focal length, principal point, and the parameters related to lens distortions. More details about the FPI camera calibration are given by Oliveira et al. (2016) and Tommaselli et al. (2019). Another important requirement is the misalignment correction between the sensors. After estimating the IOPs, the boresight angles were determined using a hypercube in the calibration field, as described by Tommaselli et al. (2013).

An aerial survey with UAV was performed with the FPI camera (Figure 4), in which an image block of 21 hypercubes distributed in three flight strips was used for the tests. This block was acquired at a flight height of $80 \mathrm{~m}$ with flying speed of $4 \mathrm{~m} / \mathrm{s}$, forward overlap of $60 \%$ and side overlap of $60 \%$, generating images with 5-cm GSD (Figure 5). In this case, the integration time was set with $5 \mathrm{~ms}$, which represents an elapsed time of $0.779 \mathrm{~s}$ to acquire an entire hypercube. In the object space, the displacement between the first and last band corresponds to $3.6 \mathrm{~m}$ (depicted in Figure 1).

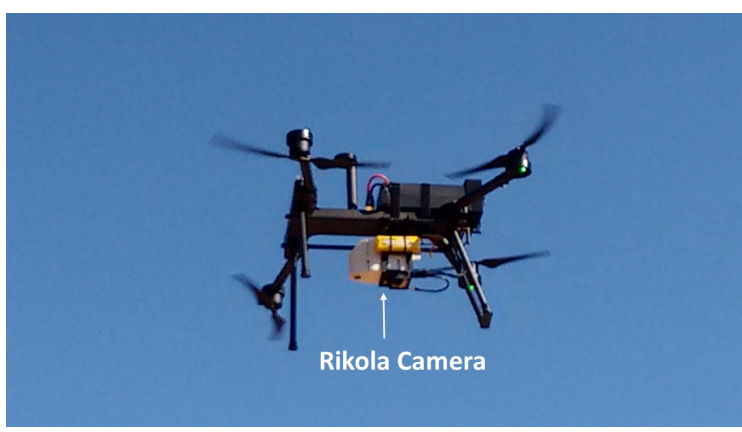

Figure 4. UAV with the FPI camera on board.

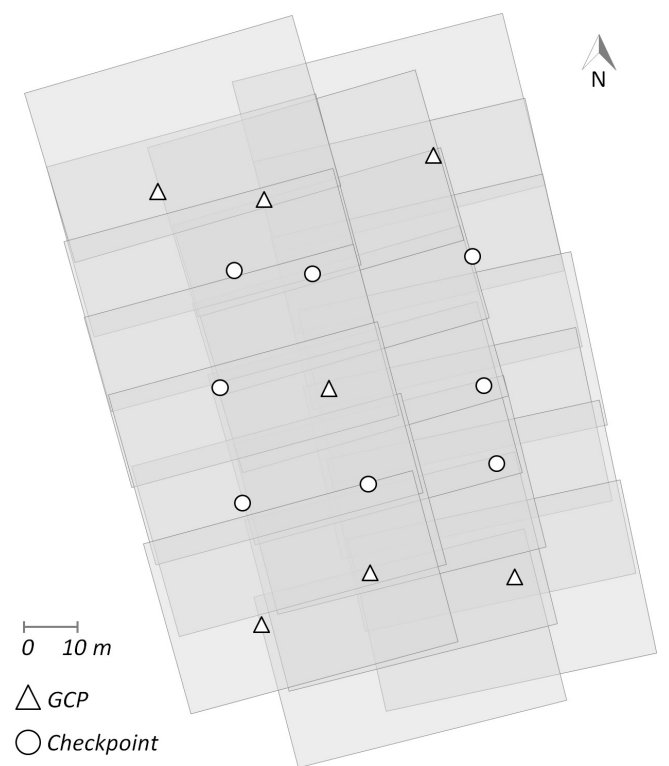

Figure 5. Hyperspectral image block with ground points used in the bundle adjustment with polynomial models. 


\subsection{Processing}

The image orientation procedure was conducted using bundle adjustment with polynomial models, in which four distributed bands were determined as samples to apply the tie point generation technique and estimate the polynomial functions.

Previously, the tie points were produced by the proposed technique using local SIFT, majority voting and transfer function followed by area-based matching with sub-pixel refinement, where only correlation coefficients higher than $70 \%$ were accepted for matches. Within the procedure of selecting reliable matches to generate the transfer functions, the goal was to find at least two matches to estimate the parameters of the transfer functions. In addition, the proposed technique was compared with the technique named M-estimator sample consensus (MSAC) (Torr and Zisserman, 2000), which is based on random sample consensus (RANSAC) and is used in match filtering procedures. MSAC is an efficient technique for filtering mismatches when there are many inliers, since there is an underlying mathematical model in which random matches should be fitted to test the solution. Three models can be used: affine, projective, or similarity (Helmert). This latter model was used for comparison with the proposed technique, considering the confidence interval of $95 \%$ and maximum distance of 2 pixels.

When few inliers are mixed with many outliers, MSAC tends to fail. In contrast, Helmert transformation with majority voting extracts reliable matches even with few inliers, since all matches are computed in the accumulator matrix. Then the dominant matches, which converge to the same bin in the matrix, are selected. In performance tests with the proposed filtering, the majority voting technique was $50 \%$ faster than the MSAC. Figure 6 shows an example of filtering in which the MSAC failed. In this case, the same set of matches presented in Figure 6(a) was filtered by both techniques. Figure 6(b) shows the result with majority voting (19 correct matches), while Figure 6(c) shows the result with MSAC (4 mismatches).

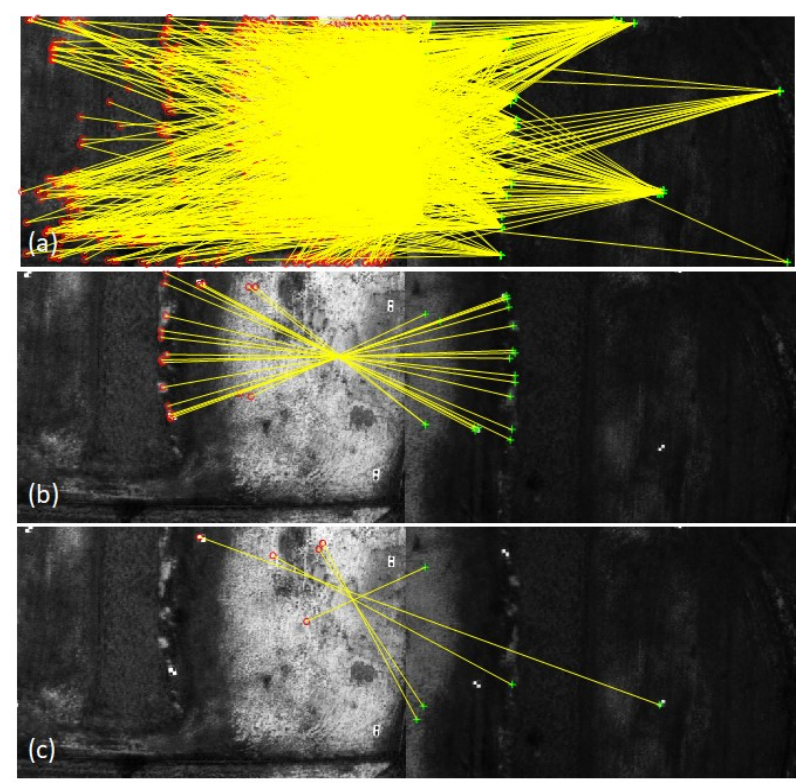

Figure 6. Match pairs between same bands of hypercubes in side strip $\left(180^{\circ}\right.$ rotation can be noticed). (a) Image matching with unfiltered matches. (b) Filtering by Helmert transformation combined with majority voting. (c) Filtering by MSAC.
To perform the image orientation by bundle adjustment with polynomials, positions of the perspective centres were obtained from the camera's GPS receiver (navigation accuracy latitude and longitude) to be used as initial approximations in the bundle adjustment. The polynomial parameters were set with weighted constraints according to the flying speed. Seven ground control points (GCPs) and eight checkpoints were also used in this procedure, as shown in Figure 5. Previously, the IOPs were calibrated as well as all image coordinates were also rectified to correct the misalignment between the sensors, as proposed by Berveglieri et al. (2019).

\subsection{Results}

The polynomial technique and a conventional bundle adjustment were applied with the same set of tie points of the four sample bands for comparison purposes. Two sets of tie points were tested. The first set was formed by the tie points generated by the proposed technique (tie points with the maximum number of rays), and the other was composed of common tie points (at least two radii).

Table 2 presents the values of root mean square error (RMSE) calculated on the checkpoints, considering both techniques with the two set of tie points. As can be seen in Table 2, the number of rays in the tie points has an impact on the resulting accuracy. When tie points appearing in the four bands and in the adjacent cubes were used (maximum number of rays), minor discrepancies were obtained in the checkpoints. In this case, both techniques (I and II) produced similar RMSEs: less than $5 \mathrm{~cm}$ (1 GSD) in XY and approximately $11 \mathrm{~cm}(2 \mathrm{GSD})$ in $\mathrm{Z}$.

\begin{tabular}{|c|c|c|c|c|}
\hline$\#$ & Technique & $\begin{array}{c}\text { RMSEx } \\
(\mathrm{m})\end{array}$ & $\begin{array}{c}\text { RMSEy } \\
(\mathrm{m})\end{array}$ & $\begin{array}{c}\text { RMSEz } \\
(\mathrm{m})\end{array}$ \\
\hline I & $\begin{array}{c}\text { Polynomial (maximum } \\
\text { number of rays) }\end{array}$ & 0.030 & 0.039 & 0.114 \\
\hline II & $\begin{array}{c}\text { Conventional bundle } \\
\text { adjustment (maximum } \\
\text { number of rays) }\end{array}$ & 0.031 & 0.040 & 0.125 \\
\hline III & $\begin{array}{c}\text { Polynomial (common } \\
\text { tie points) }\end{array}$ & 0.063 & 0.070 & 0.200 \\
\hline IV & $\begin{array}{c}\text { Conventional bundle } \\
\text { adjustment (common } \\
\text { tie points) }\end{array}$ & 0.055 & 0.072 & 0.182 \\
\hline
\end{tabular}

Table 2. RMSE resulting from the checkpoints.

In comparison (III and IV), when conventional tie points extraction techniques were used, it cannot be ensured that corresponding points appear in the four bands and, thus, larger discrepancies were obtained in the checkpoints: approximately $7 \mathrm{~cm}(1.4 \mathrm{GSD})$ in XY and $20 \mathrm{~cm}$ in $\mathrm{Z}(\sim 4 \mathrm{GSD})$. The increase in the discrepancy in XY can be explained by the short baseline that, in many cases, results from pairs of tie points between very close bands. This produces a weak geometry for the intersection of rays in the object space. Consequently, the error in $\mathrm{Z}$ has more impact, as reported in Table 2 when using common tie points.

In summary, the overall accuracy was similar between both techniques in the two comparisons presented (I with II, III with IV). However, the EOPs estimated with the conventional bundle adjustment do not produces the expected trajectory in the hypercube because they do not have a geometric constraint, which hampers the interpolation for other bands. In contrast, the polynomial technique uses a model in 
which observations and estimated EOPs are fitted to a mathematical model and then enables interpolation of other bands according to the parameters of the polynomial function. Figure 7 shows the effect of the adjustment in a hypercube when bands are interpolated by the polynomial technique in comparison with 25 bands directly estimated by conventional bundle adjustment. In the polynomial technique, the 25 bands were interpolated from the four sample bands used to estimate the polynomial parameters. As it can be seen, the ordinary bundle adjustment results in scattered band positions that do not follow a geometric model. Therefore, intermediate bands cannot be accurately interpolated.

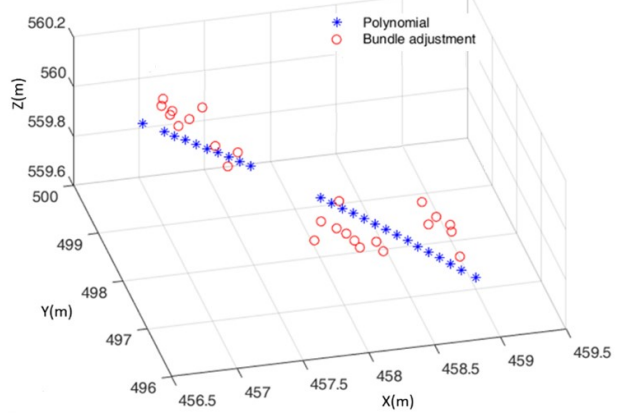

Figure 7. Twenty-five band positions of a hypercube after estimation by both techniques.

\section{CONCLUSION}

In this study, a tie point generation technique, in which tie points are extracted on all sample bands was presented, tested and compared. The technique results in points with the maximum number of rays, improving the geometric robustness for the image orientation procedure.

Typically, hyperspectral images produces lower number of tie points in comparison with RGB images, due to low contrast, lower resolution, and noise. Due to these imaging characteristics and the polynomial approach, the tie point generation technique was developed with transfer functions and correlation analysis. The objective was to ensure points distributed in the images and appearing in all sample bands. In the selection step of reliable matches, used to define transfer functions, the Helmert transformation combined with majority voting technique was more efficient than the MSAC technique, when few inliers are available. Such tie points were generated to support the image orientation technique using bundle adjustment with polynomial models, which enables a better geometric fit of the polynomial models using four sample bands of 25-band hypercubes. From the estimated polynomial parameters, all 25 bands were interpolated. In contrast, the orientation of images by conventional bundle adjustment does not allow interpolation of bands from sample bands, because it does not have a geometric fitting model for band displacement.

Based on the experiments, both ordinary bundle adjustment and polynomial techniques obtained similar RMSEs. Both techniques demonstrated that the geometric robustness of the generated tie points improved the results. They achieved an accuracy of 1 GSD in planimetry and 2 GSD in altimetry. In future works, experiments will be developed to assess the generation of hiperespectral orthomosaics.

\section{ACKNOWLEDGEMENTS}

The research performed in this study was supported by the Conselho Nacional de Desenvolvimento Científico $e$ Tecnológico (CNPq) [Grant 150306/2018-0] and the São Paulo Research Foundation (FAPESP) [Grant 2014/05533-7]

\section{REFERENCES}

Aasen, H., Burkart, A., Bolten, A., Bareth, G., 2015. Generating 3D hyperspectral information with lightweight UAV snapshot cameras for vegetation monitoring: From camera calibration to quality assurance. ISPRS Journal of Photogrammetry and Remote Sensing 108, 245-259. http://dx.doi.org/10.1016/j.isprsjprs.2015.08.002

Berveglieri, A., Tommaselli, A.M.G., 2019. Geometric filtering of matches between points in bands of hyperspectral cubes. IEEE Geoscience and Remote Sensing Letters 16, 492-496. https://doi.org/10.1109/LGRS.2018.2874178

Berveglieri, A., Tommaselli, A.M.G., Honkavaara, E., 2017. Estimating exterior orientation parameters of hyperspectral bands based on polynomial models. ISPRS - International Archives of the Photogrammetry, Remote Sensing and Spatial Information Sciences XLII-3/W3, 19-25. https://doi.org/10.5194/isprs-archives-XLII-3-W3-19-2017

Berveglieri, A., Tommaselli, A.M.G., Santos, G., Santos, L.D., Honkavaara, E., 2019a. Performance evaluation of sequential band orientation by polynomial models in hyperspectral cubes collected with UAV. ISPRS - International Archives of the Photogrammetry, Remote Sensing and Spatial Information Sciences XLII-2/W13, 1625-1629. https://doi.org/10.5194/isprs-archives-XLII-2-W13-1625-2019

Berveglieri, A., Tommaselli, A.M.G., Santos, L.D., Honkavaara, E., 2019b. Bundle adjustment of a time-sequential spectral camera using polynomial models. IEEE Transactions on Geoscience and Remote Sensing 1-12. https://doi.org/10.1109/TGRS.2019.2925783

Honkavaara, E., Rosnell, T., Oliveira, R., Tommaselli, A., 2017. Band registration of tuneable frame format hyperspectral UAV imagers in complex scenes. ISPRS Journal of Photogrammetry and Remote Sensing 134, 96-109. https://doi.org/10.1016/j.isprsjprs.2017.10.014

Jakob, S., Zimmermann, R., Gloaguen, R., 2017. The need for accurate geometric and radiometric corrections of drone-borne hyperspectral data for mineral exploration: MEPHySTo-a toolbox for pre-processing drone-borne hyperspectral data. Remote Sensing 9. https://doi.org/10.3390/rs9010088

Kenefick, J.F., Gyer, M.S., Harp, B.F., 1972. Analytical selfcalibration. Photogrammetric Engineering 38, 1117-1126.

Lowe, D.G., 2004. Distinctive image features from scaleinvariant keypoints. International Journal of Computer Vision 60, 91-110. https://doi.org/10.1023/B:VISI.0000029664.99615.94

Mikhail, E.M., Bethel, J.S., McGlone, C.J., 2001. Introduction to modern photogrammetry. John Wiley \& Sons Inc., New York. 
Moriya, É.A.S., Imai, N.N., Tommaselli, A.M.G., Miyoshi, G.T., 2017. Mapping mosaic virus in sugarcane based on hyperspectral images. IEEE Journal of Selected Topics in Applied Earth Observations and Remote Sensing 10, 740-748. https://doi.org/10.1109/JSTARS.2016.2635482

Oliveira, R.A., Tommaselli, A.M.G., Honkavaara, E., 2019. Generating a hyperspectral digital surface model using a hyperspectral 2D frame camera. ISPRS Journal of Photogrammetry and Remote Sensing 147, 345-360. https://doi.org/10.1016/j.isprsjprs.2018.11.025

Oliveira, R.A., Tommaselli, A.M.G., Honkavaara, E., 2016. Geometric calibration of a hyperspectral frame camera. Photogrammetric Record 31, 325-347. https://doi.org/10.1111/phor.12153

Senop Ltd., 2017. Hyperspectral camera. URL http://senop.fi/en/optronics-hyperspectral (accessed 10.20.19).

Tommaselli, A.M.G., Galo, M., Moraes, M.V.A., Marcato Junior, J., Caldeira, C.R.T., Lopes, R.F., 2013. Generating virtual images from oblique frames. Remote Sensing 5, 18751893.

Tommaselli, A.M.G., Santos, L.D., Oliveira, R.A. de, Berveglieri, A., Imai, N.N., Honkavaara, E., 2019. Refining the interior orientation of a hyperspectral frame camera with preliminary bands co-registration. IEEE Journal of Selected Topics in Applied Earth Observations and Remote Sensing 12, 2097-2106. https://doi.org/10.1109/JSTARS.2019.2911547

Torr, P.H.S., Zisserman, A., 2000. MLESAC: A new robust estimator with application to estimating image geometry. Computer Vision and Image Understanding 78, 138-156. https://doi.org/10.1006/cviu.1999.0832 